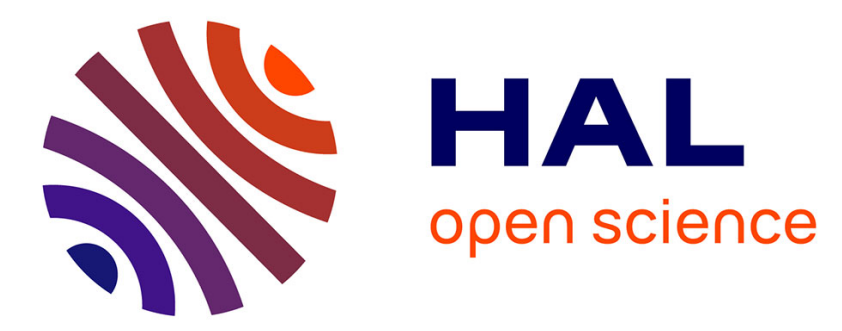

\title{
Transient Quantum Trapping of Fast Atoms at Surfaces
} M. Debiossac, A. Zugarramurdi, P. Lunca-Popa, A. Momeni, H. Khemliche, A. g. Borisov, P. Roncin

\section{To cite this version:}

M. Debiossac, A. Zugarramurdi, P. Lunca-Popa, A. Momeni, H. Khemliche, et al.. Transient Quantum Trapping of Fast Atoms at Surfaces. Physical Review Letters, 2014, 112 (2), pp.023203. 10.1103/PhysRevLett.112.023203 . hal-01417290

\section{HAL Id: hal-01417290 \\ https://hal.science/hal-01417290}

Submitted on 12 Oct 2021

HAL is a multi-disciplinary open access archive for the deposit and dissemination of scientific research documents, whether they are published or not. The documents may come from teaching and research institutions in France or abroad, or from public or private research centers.
L'archive ouverte pluridisciplinaire HAL, est destinée au dépôt et à la diffusion de documents scientifiques de niveau recherche, publiés ou non, émanant des établissements d'enseignement et de recherche français ou étrangers, des laboratoires publics ou privés. 


\title{
Transient Quantum Trapping of Fast Atoms at Surfaces
}

\author{
M. Debiossac, ${ }^{1}$ A. Zugarramurdi, ${ }^{1}$ P. Lunca-Popa, ${ }^{1}$ A. Momeni, ${ }^{1,2}$ H. Khemliche, ${ }^{1}$ A. G. Borisov, ${ }^{1,}{ }^{*}$ and P. Roncin ${ }^{1}$ \\ ${ }^{1}$ Institut des Sciences Moléculaires d'Orsay, UMR 8214 CNRS-Université Paris-Sud, Bât. 351, \\ Université Paris-Sud, 91405 Orsay CEDEX, France \\ ${ }^{2}$ Université de Cergy-Pontoise, 33 Boulevard du Port, F-95031 Cergy, France
}

(Received 23 October 2013; published 14 January 2014)

\begin{abstract}
We report on the experimental observation and theoretical study of the bound state resonances in fast atom diffraction at surfaces. In our studies, the ${ }^{4} \mathrm{He}$ atom beam has been scattered from a high-quality $\mathrm{LiF}(001)$ surface at very small grazing incidence angles. In this regime, the reciprocal lattice vector exchange with the surface allows transient trapping of the $0.3-0.5 \mathrm{keV}$ projectiles into the quasistationary states bound by the attractive atom-surface potential well which is only $10 \mathrm{meV}$ deep. Analysis of the linewidths of the calculated and measured resonances reveals that prior to their release, the trapped projectiles preserve their coherence over travel distances along the surface as large as $0.2 \mu \mathrm{m}$, while being in average only at some angstroms in front of the last atomic plane.
\end{abstract}

DOI: 10.1103/PhysRevLett.112.023203

PACS numbers: $34.35 .+\mathrm{a}, 34.50 . \mathrm{Cx}, 68.49 . \mathrm{Bc}$

The observation [1] of thermal helium diffraction at a $\mathrm{LiF}(001)$ surface demonstrated that the particle-wave duality also applies to complex objects, thereby founding modern quantum physics. Soon after [2] it was realized that the singularities in the otherwise smooth evolution of the specular diffracted intensities could be related to the region of few angstroms above the surface plane, where the attractive polarization forces induce a potential well accommodating few bound states [3-6]. The so-called bound state resonances (BSRs) [4-11] emerge in diffraction patterns when the reciprocal lattice vector exchange with the surface brings the projectile atom into a bound state, where it remains trapped until subsequent reciprocal lattice vector exchange. The lattice-assisted transition between the propagating continuum state and the bound state is also behind the operation of, e.g., grating couplers used to excite surface plasmons or to match the waveguide mode with incident light [12-14]. Owing to their narrow width, the BSRs offer a high-resolution window into the atom-surface interaction dynamics in the van der Waals regime, challenging the theoretical descriptions [15] of weakly bound systems.

The above studies of the BSRs were performed at thermal or hyper-thermal energies because diffraction works best when the probe de Broglie wavelength $\lambda$ is comparable with lattice period. As a second constraint, specific to the atom-surface interactions, $\lambda$ should remain larger than the size of typical imperfections such as atomic displacements due to thermal vibrations, otherwise the phase information is lost. This decoherence source known as the Debye-Waller factor forced the use of projectile energies below $100 \mathrm{meV}$ and usually low crystal temperatures. However, it has been recently demonstrated [16-18] that with grazing incidence geometry the fast atom diffraction (FAD or GIFAD) at surfaces is possible for scattering of $\mathrm{keV}$ atoms despite $\lambda$ is in the $\mathrm{pm}$ range.
This can be understood as due to the quasidecoupling of the swift motion parallel to the surface and slow motion perpendicular to the surface, where the latter is characterized by the wavelength back in the $\AA$ range. In addition, the decoherence of the atom beam can be made low by setting optimum scattering conditions [19,20,21]. By now, the FAD has been observed for a variety of surfaces [22-27], offering a table-top surface analysis technique.

In this letter we report theoretical investigation and tour de force $\mathrm{FAD}{ }^{4} \mathrm{He} / \mathrm{LiF}(001)$ experiments allowing us to reveal for the first time the BSRs in the keV energy range. While the effect of the BSRs on the diffraction is similar to that observed 80 years ago [2] with thermal energy beams, the very fact of their existence in the $\mathrm{keV}$ projectile energy range was highly hypothetical. Indeed, the energy differences between the BSRs are in the $\mathrm{meV}$ range. Then, from the time to energy uncertainty principle it follows that for the BSRs to be resolved, the trapped fast $\mathrm{He}$ atoms have to coherently travel over distances of $10^{3} \AA$, and this is while being only at some $\AA$ in front of the last atomic layer. Even though we are using an $\operatorname{LiF}(001)$ target with wide band gap, the possibility to reduce the electron and phonon excitations and to preserve the coherence is far from being obvious in such conditions. This is without saying that at such a fast motion parallel to the surface any surface defect could be a strong enough perturbation to destroy the coherence. The observation of the BSRs allows an unprecedented access to the details of the interaction of swift projectiles with surfaces including the target excitation probabilities.

In our experiments, ${ }^{4} \mathrm{He}$ atom beams with energy $E_{0}=$ $0.2-0.5 \mathrm{keV}$ are grazingly incident at $\mathrm{LiF}(001)$ surface at polar angle $\Phi$, as sketched in Fig. 1(a). The beam is oriented at small azimuthal misalignment angle $\Gamma$ with respect to the $\langle 110\rangle$ low index direction defined as $y$ axis. 

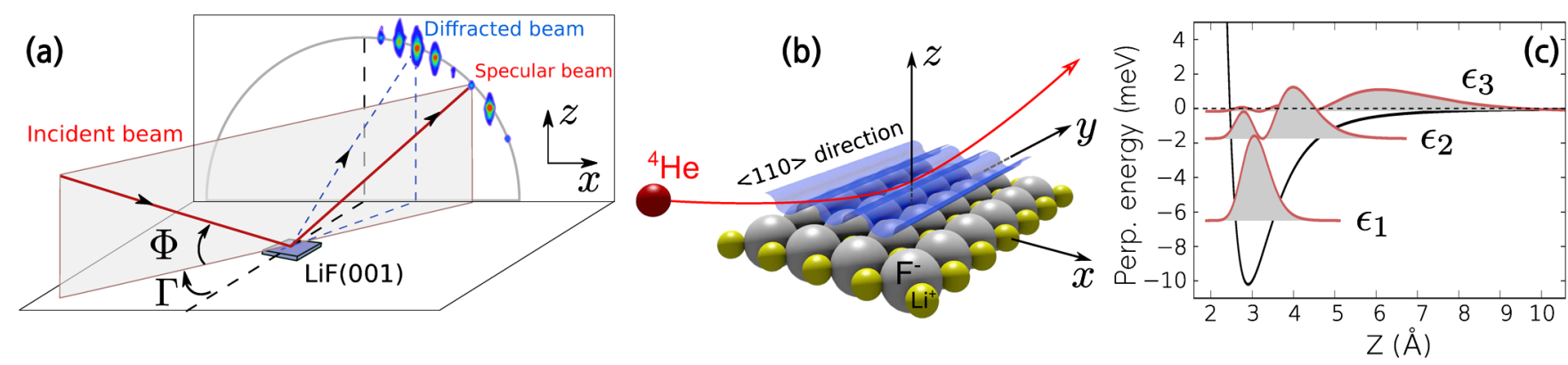

FIG. 1 (color online). (a) Sketch of the experiment. The ${ }^{4} \mathrm{He}$ atoms are incident at the $\mathrm{LiF}(001)$ surface at grazing polar $\Phi$ and azimuthal misalignment $\Gamma$ angle measured from the $\langle 110\rangle$ direction ( $y$ axis). The diffraction image corresponds to the actual data and shows well-resolved diffraction spots comparable with the incident beam profile. (b) Atomistic view of the LiF(001) surface and schematic trajectory of the projectile in the projectile-surface interaction potential averaged along the $\langle 110\rangle$ axial channel. (c) The attractive part of the $\mathrm{He} / \mathrm{LiF}$ interaction potential averaged in the surface plane is shown as function of the $z$-coordinate perpendicular to the surface. We also show the probability densities of the first three ${ }^{4} \mathrm{He}$ bound states.

The perpendicular to the surface motion of the incident projectiles is slow, with corresponding momentum $k_{\perp}=$ $k \sin \Phi$ and energy $E_{\perp}=k_{\perp}^{2} / 2 M$. Here, $M$ is the projectile mass, and $k=\sqrt{2 M E_{0}}$ is the total momentum. The motion parallel to the surface is fast with momentum component $k_{\|} \sim k$. Upon scattering, the projectiles might exchange a reciprocal lattice vector with the surface $\mathbf{k}_{\|} \rightarrow \mathbf{k}_{\|}+$ $m \hat{e}_{x} G+n \hat{e}_{y} G$, where $G=1.170 a_{0}^{-1}$, and $\hat{e}_{x}\left(\hat{e}_{y}\right)$ is the unit length vector along the $x(y)$ axis defined in Fig. 1(a) and Fig. 1(b).

It has been shown $[20,28-30]$ that for small misalignment $\Gamma$, the fast projectiles effectively "feel" the potential averaged along the low index (here $\langle 110\rangle$ ) direction as sketched in Fig. 1(b). The only effective reciprocal lattice vector exchange is along the $x$ axis across atomic rows forming the axial channel, i.e., with $n=0$. From the total energy conservation, for small $\Phi$ and $\Gamma$, the perpendicular to the surface motion of the diffracted particles is associated with an energy

$$
E_{\perp}^{\prime}=E_{\perp}-(m G)^{2} / 2 M-m G k \Gamma / M .
$$

The $E_{\perp}^{\prime}=0$ sets the threshold for the opening of the diffraction into the $(m, n=0) \equiv m$ diffraction order. For $E_{\perp}^{\prime}>0$ the corresponding diffracted beams located on a single Laue circle can leave the surface.

The most interesting case for the present discussion is when $E_{\perp}^{\prime}<0$ and the corresponding diffraction order is closed. As known from the He atom scattering at thermal energies (TEAS) [4-11], the ${ }^{4} \mathrm{He} / \mathrm{LiF}(001)$ potential posses a slightly corrugated shallow (10 meV deep) attractive potential well, which is located at $3 \AA$ with respect to the last atomic layer as sketched in Fig. 1(c). This well hosts four bound states denoted by the quantum number $j=1, \ldots, 4$ and corresponding to the quantized motion perpendicular to the surface with negative energy $\epsilon_{j}$ [31]. The motion parallel to the surface is quasifree [32].
When $E_{\perp}$ of the incident projectiles matches the resonance condition

$$
E_{\perp} \equiv E_{\perp}^{m, j}=\epsilon_{j}+(m G)^{2} / 2 M+m G k \Gamma / M,
$$

one obtains $E_{\perp}^{\prime}=\epsilon_{j}$. As a result of the reciprocal lattice vector exchange, the He atom is trapped in the bound state above the surface and moves parallel to it. The trapped states are quasistationary, since the projectile can again exchange a reciprocal lattice vector with the surface, and escape into an open diffraction order. The BSRs manifest themselves as sharp features in the $E_{\perp}$ or $\Gamma$ dependence of the diffraction probabilities some $\mathrm{meV}$ below the thresholds for the opening of the diffraction orders [4-11].

In a quest for the signatures of BSRs in FAD we used the experimental setup described in earlier publications $[23,29]$. In brief, atomic projectiles are obtained from the primary $\mathrm{He}^{+}$ions extracted from a commercial ion gun, and neutralized in an effusive gas cell. The resulting fast atom beam is sharply collimated so that the beam divergence angle of $180 \mu \mathrm{rad}$ is reached. Experiments are performed in ultrahigh vacuum conditions at a base pressure of some $10^{-11}$ mbar. The atoms diffracted by the surface hit a microchannel plate amplifier stacked onto a phosphor screen located $740 \mathrm{~mm}$ downstream. The diffraction pattern is captured online by a CCD camera with a resolution comparable to that of the beam divergence. The $\operatorname{LiF}(001)$ sample was cleaved in air and rapidly introduced into the UHV chamber for the FAD measurements [33]. An annealing to $500 \mathrm{~K}$ is sufficient to allow observation of intense and well-resolved diffraction patterns. By varying the $\Phi$ angle and the total energy of the beam we could sample the $E_{\perp}$ range from some meV up to some hundreds of meV. Simultaneously, the data have been taken upon variation of the misalignment angle $\Gamma$ offering more flexibility in tuning into the resonance condition [see Eq. (2)]. 


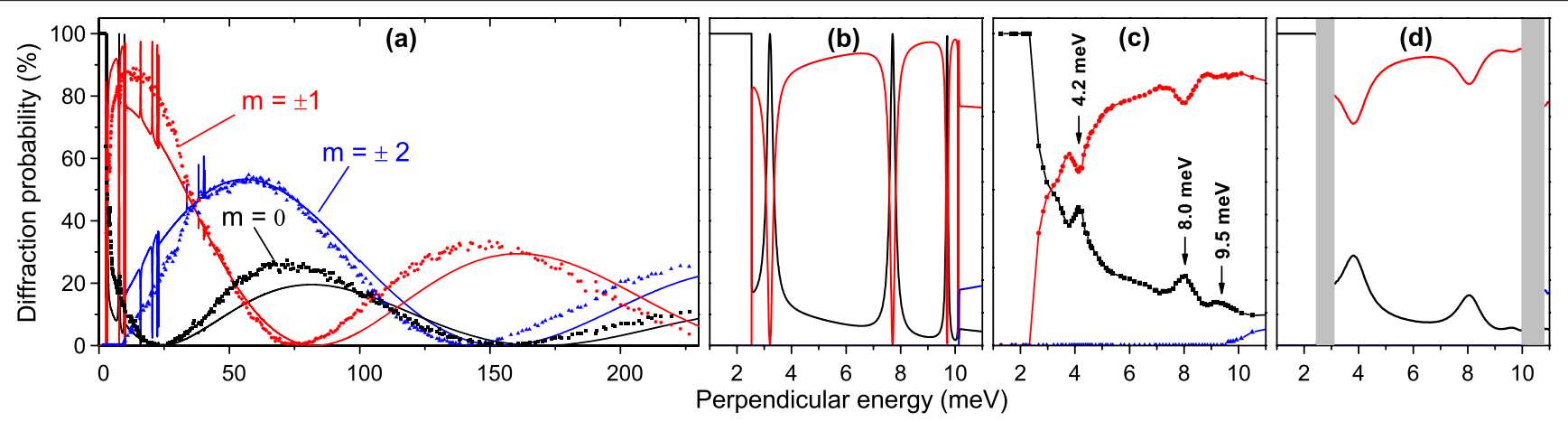

FIG. 2 (color online). Comparison between experimental (dots) and theoretical (lines) diffraction curves for the beam incident along the $\langle 110\rangle$ direction. The diffraction probabilities $\mathcal{R}_{m}$ are shown as function of the $E_{\perp}$ energy for the three lowest diffraction orders. Results for the specular reflection $(m=0)$ are shown in black. For nonspecular diffraction we sum the equal $\pm m$ probabilities: $\mathcal{R}_{-1}+\mathcal{R}_{1}$ (red); $\mathcal{R}_{-2}+\mathcal{R}_{2}$ (blue). Panel (a) covers large $E_{\perp}$ energy scale. In panels (b), (c) the low-energy region is zoomed for the theoretical (b) and experimental (c) data. The energies of the experimentally observed structures are indicated in panel (c). Panel (d) shows the WPP results accounting for the BSRs decoherence rate of $1 \mathrm{meV}$. The WPP method does not apply close to the diffraction thresholds so that the corresponding energy intervals are shaded in figure.

Theoretical calculations of FAD have been performed with the time-domain wave packet propagation (WPP) method detailed elsewhere $[17,28,29]$. We also used the stationary approach [34] based on the extraction of the scattering matrix [35] from the solution of the closecoupling equations [36]. The projectile-surface interaction potential has been set such that it is appropriate for both small and large perpendicular energies of the incident beam. To this end we took as a basis the low-energy part of the potential as proposed for TEAS with helium atoms $[4,5,6]$. It incorporates the shallow attractive well in front of the surface shown in Fig. 1(c) and allows us to reproduce the energies of the BSRs [31]. The high-energy part of the potential has been adopted from the earlier studies of $\mathrm{He} / \mathrm{LiF}(001)$ FAD $[17,28,29]$, and the surface rumpling [37] has been also included. The two potentials were merged such that the FAD data are retrieved over a broad $E_{\perp}$ energy range.

In Fig. 2 we present the experimental and theoretical $E_{\perp}$ energy dependence of the diffraction probabilities $\mathcal{R}_{m}$ for the ${ }^{4} \mathrm{He} F A D$ at $\mathrm{LiF}(001)$ surface. The incident beam is aligned along the $\langle 110\rangle$ direction $(\Gamma=0)$. The diffraction probability $\mathcal{R}_{m}$ is defined as $\mathcal{R}_{m}=\mathcal{J}_{m} / \sum_{\ell} \mathcal{J}_{\ell}$, where $\mathcal{J}_{\ell}$ is the intensity of the $\ell$ th diffracted beam and the sum runs over all open diffraction orders. The panel (a) of the figure covers large $E_{\perp}$ range, where good agreement between calculated and measured data is reached. In overall, the well documented $\mathcal{R}_{m}\left(E_{\perp}\right)$ dependence is retrieved, which can be described by the Bessel functions within the repulsive hard wall model $[17,18]$. However, at low perpendicular energies the scattering is affected by the shallow attractive potential well. The description based on the fully repulsive potential breaks down, and sharp resonant features emerge particularly clear in the theoretical curves.

The panels (b), (c), and (d) of Fig. 2 zoom at the low perpendicular energy range. Here, only specular reflection
( $m=0$ ) is possible for $E_{\perp}<2.3 \mathrm{meV}$, the first diffraction order opens at $E_{\perp}=2.3 \mathrm{meV}$, and the second diffraction order opens at $E_{\perp}=10.1 \mathrm{meV}$. Because of the small number of the open diffraction orders, transient trapping of the projectiles leads to the well contrasted resonant features. Consistent with four bound states of ${ }^{4} \mathrm{He}$ in the attractive well of the atom-surface interaction potential [31], theoretical calculations shown in Fig. 2(b) predict four $j=1, \ldots, 4$ narrow resonances in the diffraction probabilities below the $m= \pm 2$ diffraction threshold. At the resonance energies given by Eq. (2) with $\Gamma=0$, and $m= \pm 2$, the first diffraction order $(m= \pm 1)$ appears suppressed because of the interference between the resonant and nonresonant contributions. Consequently, the specular reflection probability rises to one. Note that the calculations also show the BSRs connected to the higherorder diffraction thresholds at $E_{\perp}>15 \mathrm{meV}$ [Fig. 2(a)]. However, the resonant variation of the diffraction probabilities is smaller in these cases because the outgoing flux is distributed among larger number of diffracted beams. Gradually, upon increasing $E_{\perp}$ the resonances become graphically unresolvable. It is worth noting that besides the BSRs, the attractive potential well manifests itself in a sharp threshold behavior of the diffraction probabilities, as has been studied in formal scattering theory and low-energy electron-molecule scattering [38,39].

The calculated BSRs are retrieved in experimental results shown in Fig. 2(c). The pronounced resonance contrast predicted by the theory led us to concentrate the experimental effort in the low $E_{\perp}$ energy range. Here, the departure of the measured diffraction curves from the Bessel-like dependence, and sharp $m= \pm 1$ threshold behavior is evident pointing out new physics in FAD. Low decoherence of the beam at small perpendicular energies [21] allows for a clear observation of the two $j=1$ and $j=2$ resonances associated with increased specular 


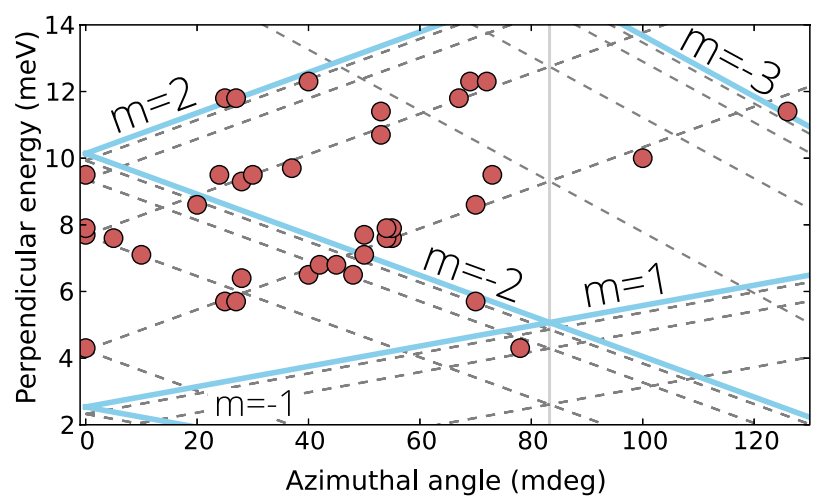

FIG. 3 (color online). $\quad E_{\perp}$ energies of the resonant structures in the diffraction curves measured with $300 \mathrm{eV}{ }^{4} \mathrm{He}$ beam. The data are shown as function of the azimuthal misalignment angle $\Gamma$. Dashed lines show the resonance energy dispersion as obtained from Eq. (2) using bound state energies from Ref. [11]. The vertical solid gray line marks the first Brillouin zone boundary at $k \Psi=G / 2$. The energy thresholds for the opening of the different diffraction orders are shown with correspondingly labeled blue lines.

reflection and reduced $m= \pm 1$ diffraction. Using Eq. (2) we determined the binding energies $\epsilon_{1,2}$ of the ${ }^{4} \mathrm{He}$ trapped states in a very good agreement with TEAS data [31]. Thus, the resonance at the incident beam energy perpendicular to the surface $E_{\perp}=4.2 \mathrm{meV}$ corresponds to $\epsilon_{1}=-5.95 \mathrm{meV}$, and the resonance at $E_{\perp}=8 \mathrm{meV}$ corresponds to $\epsilon_{2}=-2.12 \mathrm{meV}$. Based on the comparison with theoretical results, we tentatively attribute a broad structure at $E_{\perp} \approx 9.5 \mathrm{meV}$ to the $j=3$ and $j=4$ BSRs. However, the nearby $m= \pm 2$ threshold and overlap of the resonance peaks hinders accurate extraction of the corresponding binding energies.

The measured resonances display larger widths and smaller variation of the diffraction probabilities as compared to the theory. The difference between the experiment and the calculation performed for the elastic scattering at perfect surface can be attributed to the (i) instrumental broadening and to the (ii) decoherence due to the target excitations and defect scattering. Indeed, because of the long interaction times with $\operatorname{LiF}(001)$ surface, the decoherence is expected to be particularly strong for the projectiles trapped in the BSRs. To illustrate the effect, we have performed WPP calculations adding a complex absorbing potential $[40,41]$ to the projectile-surface interaction. As follows from Fig. 2(d), inclusion of the loss of coherent particles allows us to approach the experimental data.

Along with the perpendicular energy $E_{\perp}$ scan for the perfectly aligned beam, an alternative procedure to reveal the BSRs consists of measuring the diffraction intensities upon variation of the azimuthal misalignment angle $\Gamma$. The $\Gamma$-angle scans for several fixed polar incidence angles $\Phi$ allow us to determine the position of the resonance features at the $\left(\Gamma, E_{\perp}\right)$ plane. In Fig. 3 the resonance energies $E_{\perp}^{m, j}$ extracted from the experimental data are shown as a function of the misalignment angle. Essentially the resonances below the $m= \pm 2$ diffraction thresholds are observed consistently with results reported in Fig. 2. The linear dispersion given by Eq. (2) with $\epsilon_{j}$ values as reported by Derry $e t$ al. $[11,31]$ nicely describes present experimental data. This further supports the conclusion that transient trapping of the projectiles into the BSRs is possible in FAD experiments despite the huge $\left(E_{0} / \epsilon_{j} \sim 5 \times 10^{4}\right)$ ratio between the total energy of the projectile and its binding energy in the potential well above the surface.

Besides the bound state energy extraction, analysis of the BSRs provides further unprecedented details on the interaction of swift projectiles with surfaces. Indeed, our theoretical calculations for the purely elastic scattering at a perfect surface predict very small resonance widths $\Delta E_{\perp} \sim 0.25 \mathrm{meV}$. These correspond to an elastic lifetime of $\tau=2.6 \mathrm{ps}$, meaning that prior to the reciprocal lattice vector exchange leading to the escape from the trapped state, the $0.3 \mathrm{keV}{ }^{4} \mathrm{He}$ atoms coherently travel a distance $L=0.3 \mu \mathrm{m}$ above the surface. The measured resonance width is $\sim 0.5 \mathrm{meV}(\sim 0.7 \mathrm{meV})$ for the resonance at $E_{\perp}=4.2 \mathrm{meV}\left(E_{\perp}=8 \mathrm{meV}\right)$ [31]. Thus, we estimate from the experimental data $L \sim 0.2 \mu \mathrm{m}$. This is a lower-bound estimate for the coherent travel distance $L$ because of the instrumental broadening effects [41].

In summary, in this experimental and theoretical joint effort we have found a clear evidence for the coherent trapping of fast $0.3-0.5 \mathrm{keV}{ }^{4} \mathrm{He}$ beams grazingly incident at the $\operatorname{LiF}(001)$ surface. By measuring resonances in the diffraction patterns, we have shown that the reciprocal lattice vector exchange with the surface leads to the trapping of the projectiles in the quasistationary states bound by the $10 \mathrm{meV}$ deep attractive well of the projectile-surface potential. This sets the ratio of the total energy to the binding energy as high as $5 \times 10^{4}$. Thus, the regime observed here is very different from the earlier studies with thermal $\mathrm{He}$ atom beams. It is also worth to mention that, while for the "skipping motion" [42] reported earlier, the $\mathrm{keV}$ ionic projectiles follow classical trajectories bound by the image charge interaction, the present process is purely quantum. The very existence of the narrow resonant structures in diffraction probabilities with a width of a fraction of $\mathrm{meV}$ requires quantum interference between resonant and nonresonant scattering. Thus, the coherence of the trapped beam is preserved over basically macroscopic travel distances of $\sim 0.2 \mu \mathrm{m}$ at some angstroms above the topmost atomic layer of the $\mathrm{LiF}(001)$ surface. Along with the high surface quality, this demonstrates that the electron and phonon excitations by the projectiles can be very low, a subject that attracts a vivid interest in the context of surface reactivity [43-46].

This work has been funded in part by the French Agence Nationale de la Recherche (No. ANR-2011EMMA-003-01). M. Debiossac and A. Zugarramurdi contributed equally to this work. 
*andrei.borissov@u-psud.fr

[1] I. Estermann and O. Stern, Z. Phys. 61, 95 (1930).

[2] R. O. Frisch and O. Stern, Z. Phys. 84, 430 (1933).

[3] J. E. Lennard-Jones and A. F. Devonshire, Nature (London) 137, 1069 (1936).

[4] V. Celli, D. Eichenauer, A. Kaufhold, and J. P. Toennies, J. Chem. Phys. 83, 2504 (1985).

[5] P. W. Fowler and J. M. Hutson, Phys. Rev. B 33, 3724 (1986).

[6] H. Hoinkes, Rev. Mod. Phys. 52, 933 (1980).

[7] H. Chow and E. D. Thompson, Surf. Sci. 59, 225 (1976).

[8] P. Cantini and R. Tatarek, Phys. Rev. B 23, 3030 (1981).

[9] J. G. Mantovani, J. R. Manson, and G. Armand, Surf. Sci. 143, 536 (1984).

[10] J. A. Meyers, and D. R. Frankl, Surf. Sci. 51, 61 (1975).

[11] G. Derry, D. Wesner, S. Krishnaswamy, and D. R. Frankl, Surf. Sci. 74, 245 (1978).

[12] K. Tiefenthaler and W. Lukosz, J. Opt. Soc. Am. B 6, 209 (1989).

[13] D. K. Gramotnev, S. I. Bozhevolnyi, Nat. Photonics 4, 83 (2010).

[14] H. A. Atwater and A. Polman, Nat. Mater. 9, 205 (2010).

[15] X. Wu, M. C. Vargas, S. Nayak, V. Lotrich, and G. Scoles, J. Chem. Phys. 115, 8748 (2001).

[16] A. Schüller, S. Wethekam, and H. Winter, Phys. Rev. Lett. 98, 016103 (2007).

[17] P. Rousseau, H. Khemliche, A. G. Borisov, and P. Roncin, Phys. Rev. Lett. 98, 016104 (2007).

[18] H. Winter and A. Schüller, Prog. Surf. Sci. 86, 169 (2011).

[19] J. Lienemann, A. Schüller, D. Blauth, J. Seifert, S. Wethekam, M. Busch, K. Maass, and H. Winter, Phys. Rev. Lett. 106, 067602 (2011).

[20] F. Aigner, N. Simonović, B. Solleder, L. Wirtz, and J. Burgdörfer Phys. Rev. Lett. 101, 253201 (2008).

[21] J. R. Manson, H. Khemliche, and P. Roncin, Phys. Rev. B 78, 155408 (2008).

[22] N. Bundaleski, H. Khemliche, P. Soulisse, and P. Roncin, Phys. Rev. Lett. 101, 177601 (2008).

[23] H. Khemliche, P. Rousseau, P. Roncin, V. H. Etgens, and F. Finocchi, Appl. Phys. Lett. 95, 151901 (2009).

[24] A. Schüller, M. Busch, S. Wethekam, and H. Winter, Phys. Rev. Lett. 102, 017602 (2009).

[25] J. Seifert, A. Schüller, H. Winter, R. Włodarczyk, J. Sauer, and M. Sierka, Phys. Rev. B 82, 035436 (2010).

[26] J. Seifert and H. Winter, Phys. Rev. Lett. 108, 065503 (2012).

[27] C. A. Ríos Rubiano, G. A. Bocan, M. S. Gravielle, N. Bundaleski, H. Khemliche, and P. Roncin, Phys. Rev. A 87, 012903 (2013).
[28] A. Zugarramurdi and A. G. Borisov, Phys. Rev. A 87, 062902 (2013).

[29] A. Zugarramurdi, M. Debiossac, P. Lunca-Popa, L. S. Alarcón, A. Momeni, H. Khemliche, P. Roncin, and A. G. Borisov, Phys. Rev. A 88, 012904 (2013).

[30] D. Farías, C. Díaz, P. Nieto, A. Salin, and F. Martín, Chem. Phys. Lett. 390, 250 (2004).

[31] All data in meV. The bound state energies of ${ }^{4} \mathrm{He}$ in front of $\mathrm{LiF}(001)$ (see also Table VIII of Ref. [6]) as measured by Meyers and Frankl [10] are $\left|\epsilon_{1}\right|=5.8, \quad\left|\epsilon_{2}\right|=2.2$, $\left|\epsilon_{3}\right|=0.6,\left|\epsilon_{4}\right|=0.1$. For the same system Derry et al. [11] report $\left|\epsilon_{1}\right|=5.9,\left|\epsilon_{2}\right|=2.46,\left|\epsilon_{3}\right|=0.78,\left|\epsilon_{4}\right|=0.21$. The bound state energies derived here from the BSRs measured along the $\langle 110\rangle$ direction are $\left|\epsilon_{1}\right|=5.95$, $\left|\epsilon_{2}\right|=2.12,\left|\epsilon_{3,4}\right| \lesssim 0.6$. The widths of the first two BSRs measured here are $\Delta_{1}=0.5, \Delta_{2}=0.7$. In our elastic theory the corresponding values for the energies are $\left|\epsilon_{1}\right|=6.45$, $\left|\epsilon_{2}\right|=2.42,\left|\epsilon_{3}\right|=0.43,\left|\epsilon_{4}\right|=0.02$. The theoretical widths of the lowest two resonances are $\Delta_{1}=0.26, \Delta_{2}=0.21$.

[32] D. J. Riley, A. P. Jardine, S. Dworski, G. Alexandrowicz, P. Fouquet, J. Ellis, and W. Allison, J. Chem. Phys. 126, 104702 (2007).

[33] D. A. Wesner and D. R. Frankl, Phys. Rev. B 24, 1798 (1981).

[34] A. Zugarramurdi and A. G. Borisov (unpublished).

[35] D. W. Norcross, and M. J. Seaton, J. Phys. B 6, 614 (1973).

[36] J. R. Manson, Theoretical Aspects of Atom-Surface Scattering, edited by E. Hulpke, Springer Series in Surface Sciences (Springer-Verlag, Berlin, 1992), Vol. 27.

[37] A. Schüller, S. Wethekam, D. Blauth, H. Winter, F. Aigner, N.Simonović, B. Solleder, J. Burgdörfer, and L. Wirtz, Phys. Rev. A 82, 062902 (2010).

[38] R. K. Nesbet, J. Phys. B 13, L193 (1980).

[39] G. A. Gallup and I. I. Fabrikant, Phys. Rev. A 75, 032719 (2007).

[40] D. Neuhasuer and M. Baer, J. Chem. Phys. 90, 4351 (1989).

[41] See Supplemental Material at http://link.aps.org/ supplemental/10.1103/PhysRevLett.112.023203 for the details on the theory and experiment.

[42] K. J. Snowdon, D. J. O'Connor, and R. J. MacDonald, Phys. Rev. Lett. 61, 1760 (1988).

[43] P. Nieto et al., Science 312, 86 (2006).

[44] G.-J. Kroes, Science 321, 794 (2008).

[45] J. I. Juaristi, M. Alducin, R. Diez Muiño, H. F. Busnengo, A. Salin, Phys. Rev. Lett. 100, 116102 (2008).

[46] N. H. Nahler, J. D. White, J. LaRue, D. J. Auerbach, and A. M. Wodtke, Science 321, 1191 (2008). 